Umwelt- und Energiestrategien in China

\title{
Erneuerbare Energien ohne energische Nachhaltigkeitspolitik
}

\author{
China verstärkt sein Engagement bei Energieeffizienz und \\ Erneuerbaren Energien. Ziele sind größere Wettbewerbsfähig- \\ keit und niedrige gesamtgesellschaftlichen Kosten. \\ Ein Bekenntnis zur Nachhaltigkeit fehlt in der Politik. \\ Ursache dafür ist auch das Fehlen von Demokratie \\ und Mitbestimmung. Von Ulrich Oberndorfer
}

\begin{abstract}
A m 28. Februar diesen Jahres verabschiedete der Nationale Volkskongress in Peking das Renewable Energy Law. Durch dessen Inkrafttreten zum ersten Januar 2006 werden die Förderung von Energieeffizienz und das Streben nach größerer Unabhängigkeit von fossilen Energieträgern in China einen vorläufigen Höhepunkt erreichen.

Sollten die ambitionierten Vorhaben des Gesetzestextes umgesetzt werden, so werden im Jahr 2010 zehn Prozent des Energieverbrauchs Chinas durch Erneuerbare Energien (EE) gedeckt, während diese im Jahr 2003 nur drei Prozent ausmachten. Als wichtigste Instrumente sieht der Plan öffentliche EE-Investitionen sowie Steuervorteile und staatlich subventionierte Kredite als finanzielle Anreize für Renewables-Produzenten vor. Die Stromanbieter im „Reich der Mitte“ werden per Gesetz zur Einspeisung der EE-Anteile verpflichtet (The People's Republic of China 2005).
\end{abstract}

\section{Boom der Erneuerbaren}

Während ein Boom der Erneuerbaren für die kommenden Jahre zu erwarten ist, sind die Fortschritte Chinas in Sachen Energieeffizienz schon jetzt deutlich sichtbar. Die Energieintensität des Landes fiel zwischen 1960 und 1997 um 60 Prozent, jährlich also um durchschnittlich vier Prozent. Die staatlichen Instrumente zur Steigerung der Energieeffizienz waren und sind vielfältig. Den Hauptanreiz für Unternehmen, ihren Energieeinsatz zu reduzieren, stellte allerdings die Einführung von echten Marktbedingungen im Energiebereich dar. Die Subventionierung unprofitabler Firmen wurde abgeschafft, die Energiepreise bewegen sich heute auf Welthandelsniveau. Daneben sorgten noch weitere Reformen in Steuer- und Preissystem für einen sparsameren Umgang mit Strom und anderen Energiequellen (Chandler et al. 2002). Mit dem Inkrafttreten eines weiteren Gesetzes im vergangenen Jahr, das für Neuwagen einen Maximalbenzinverbrauch vorschreibt, hat die Volksrepublik eine weitere Stufe in Richtung Ressourcenschonung genommen und sogar für höher entwickelte Länder neue Maßstäbe gesetzt.

Der seit etwa drei Jahrzehnten andauernde Trend hin zu mehr Energieeffizienz sowie die neue EE-Welle sind für China aus wirtschaftlichen und ökologischen Gründen dringend notwendig. Bereits heute ist die Volksrepublik zweitgrößter Energiekonsument der Erde, und Prognosen lassen erwarten, dass sie bis zum Jahr 2050 auch die USA überholt haben wird. Diese erhöhte Binnennachfrage, verbunden mit rapide gestiegenen Strompreisen und akuten Engpässen in der Energieversorgung, machen in den Regionen Chinas, wo die Bedingungen für Windkraft oder Solarstromerzeugung besonders günstig sind, Erneuerbare profitabel. Dieser Effekt wird zudem durch die derzeit generell steigenden Weltmarktpreise von Öl und Gas unterstützt, die nicht zuletzt auch vom Nachfrageschock aus Fernost herrühren.

\section{Fortschritt durch höhere Preise}

Die Preissteigerungen erklären auch die Fortschritte bei der Energieeffizienz, die unternehmerischem Kalkül zurechenbar sind und nicht durch Wirtschaftsreformen, sondern durch Kostendruck induziert wurden. Dieses Argument ist besonders vor dem Hintergrund stichhaltig, dass die inländischen Produzenten trotz der in den vergangenen Jahren stark verringerten Energieintensität in diesem Bereich weiterhin Nachholbedarf besitzen: In chinesischen Produkten steckt immer noch 40 Prozent mehr Energie als in vergleichbaren Gütern, die in höher entwickelten Ländern hergestellt werden (Chandler et al. 2002). Die Anbindung an den Weltmarkt macht nachhaltige Energiestrategien also unumgänglich, wollen das Land und seine Unternehmen profitabel bleiben und größere Energieengpässe verhindern.

Abgesehen von diesen wirtschaftlichen Zwängen befinden sich die externen Kosten der konventionellen Energieproduktion in China auf höchstem Niveau. Bei deren Internalisierung wird deutlich, dass es für die Zentralregierung keine Alternative $\mathrm{zu}$ einer nationalen Energiewende gab und gibt. Vor allem der Prozess der Kohleverstromung ist verantwortlich dafür, dass das Land unter enormen Umweltbelastungen zu leiden hat, wie sie in ökonomisch entwickelten Ländern längst der Vergangenheit angehören. Dabei werden auf diese Weise noch immer zwei Drittel des chinesischen Energiebedarfs produziert (Chandler/ Gwin 2004). Die Verbrennung von aus Wassermangel häufig ungewaschener Kohle in veralteten Kraftwerken sorgt dafür, dass vor allem in Städten Grenzwerte bezüglich der Luftverschmutzung seit Jahren überschritten werden. Zudem fällt saurer Regen auf etwa 30 Prozent der Fläche Chinas. Schätzungen zufolge verursacht alleine dieser saure Regen in Wäldern, der Landwirtschaft und für das Gesundheitssystem Kosten von über 13 Milliarden Dollar pro Jahr. Um die Luftverschmutzung in den am stärksten betroffenen Gebieten des Landes zu reduzie- 
ren, muss die Zentralregierung in den kommenden Jahren ebenfalls einen Dollarbetrag in zweistelliger Millionenhöhe aufwenden (AsrlA 2003). Die langjährige Nachlässigkeit in Umweltschutzfragen kommt die Volksrepublik also teuer zu stehen. Ein neues Konzept zur Energiepolitik, das die von der Stromerzeugung verursachten gesamtgesellschaftlichen Kosten berücksichtigt, müsste daher nachhaltigen Ansätzen Rechnung tragen.

\section{Fehlende Nachhaltigkeits- strategie}

Die hauptsächlich im Energiebereich angesiedelten Maßnahmen, die in Richtung Nachhaltigkeit im Allgemeinen oder Umweltschutz im Speziellen weisen, dürfen aber nicht über das Fehlen eines breiten Bekenntnisses zur Nachhaltigkeit hinwegtäuschen. Energie- und Umweltpolitik sind nicht Teil einer ganzheitlichen nationalen Nachhaltigkeitsstrategie Chinas. Vielmehr werden bedeutende Aspekte einer in sich geschlossenen Nachhaltigkeitspolitik vom Gesetzgeber und der Administration schlicht ignoriert.

In einer jüngst veröffentlichten Studie rät die Schweizer Bank Sarasin (2004) daher nachhaltig gesinnten Investoren, Unternehmen mit signifikantem ChinaEngagement grundsätzlich mit Vorsicht zu betrachten. Hauptkritikpunkte am chinesischen Wirtschaftssystem sind dabei die laxe Sozialgesetzgebung und mangelnde Mitbestimmung in Unternehmen. Unabhängige Gewerkschaften sind nicht zugelassen, Interessensvertretung von und für Arbeitnehmer kaum möglich. Vor allem Staatsunternehmen untergraben selbst die minimalen geltenden Sozialstandards und bestimmen damit den Wettbewerbsrahmen auch für ausländische Firmen.

Obwohl aufgrund der erwähnten Probleme in den vergangenen Jahren eine Reihe von Gesetzen zur Forcierung des Umweltschutzes und von Erneuerbaren in Kraft getreten ist, ist der Fortschritt auch in diesen Bereichen bislang überschaubar. Vor allem in ländlich geprägten Provinzen wird die Regulierung zugunsten der Natur nur langsam umgesetzt oder ganz missachtet. Dies trifft auch auf den Energiebereich zu. Die Verbreitung von Erneuerbaren leidet zudem unter einer kaum entwickelten einheimischen Industrie für Turbinen sowie unter Schwierigkeiten, vor die ausländischen EE-Firmen im Land gestellt werden. Diese beklagen bürokratische Hemmnisse, rechtliche Probleme sowie mangelnden Sachverstand bei den chinesischen Behörden (Haum 2004). Zugute halten muss man China, dass sowohl die heutigen Hochtechnologiestaaten als auch die meisten Entwicklungsländer während ihrer Industrialisierung Nachhaltigkeitsanliegen in der Regel völlig ignorierten oder immer noch ignorieren. Dennoch, die Umweltpolitik des bevölkerungsreichsten Landes der Erde hat seit Jahren globale Konsequenzen. Dass diese von der Regierung aufgrund eines rein nationalen Kalküls kaum beachtet werden, ist etwa daran abzulesen, dass inzwischen nur noch die USA mehr Kohlendioxid emittieren als China (Chandler/Gwin 2004).

\section{Kein Druck von unten}

Dass trotz des offensichtlichen Nachholbedarfs in Sachen Nachhaltigkeit die staatlichen Bemühungen diesbezüglich widersprüchlich bis kaum vorhanden sind, kann zum Teil mit polit-ökonomischen Argumenten begründet werden. Soziale und ökologische Reformen, die zumindest kurzfristig wirtschaftlich Nachteile mit sich bringen, sind in den meisten westlichen Demokratien vor allem auf Initiativen von Interessensgruppen, beispielsweise von Naturschutzverbänden und Umweltinitiativen, oder den Wähler selbst zurückzuführen. In der Volksrepublik existiert dieser Druck „von der Straße" nicht. Die Regierung ist nicht auf mehrheitliche Zustimmung durch die chinesischen Bürger angewiesen. Sie kann somit wirtschafts-, sozial- und umweltpolitische Entscheidungen weitgehend losgelöst vom Volkswillen durchsetzen - abgesehen davon, dass es in China auch aufgrund der politischen Struktur keine Umweltbewegung gibt, wie sie beispielsweise im Deutschland der siebziger und achtziger Jahre entstanden ist.
Fazit

Soziale Entwicklungen, die einem rapiden Wirtschaftswachstum entgegenstehen könnten, sind von der chinesischen Führung nicht gewollt. In Richtung Umweltschutz gehende Reformen resultieren häufig, wie im Falle von EE oder Energieeffizienz, aus Bemühungen um höhere internationale Wettbewerbsfähigkeit oder aus stark gestiegenen gesellschaftlichen Kosten der Umweltverschmutzung. Darüber hinaus sind Initiativen zur Nachhaltigkeit jedoch Mangelware. $\leftarrow$

\section{Literatur \\ Association for Sustainable and Responsible Investment in China (AsrlA) (Hrsg.): China: The Investment Agenda for Building an Environmentally Sustainable Economy. Hongkong 2003. \\ Bank Sarasin (Hrsg.): „Made in China“ - Ist dieses Label nachhaltig? Basel 2004. \\ Chandler, W.; Gwin, H.: China's Energy and Emissions: A Turning Point? Pacific Northwest National Laboratory, Richland 2004. \\ Chandler, W.; Schaeffer, R.; Dadi, Z.; Shukla, P.R.; Tudela, F.; Davidson, O.; Alpan-Atamer, S.: Climate Change Mitigation in Developing Countries. Pew Center on Global Climate Change, Arlington 2002. \\ Haum, R.: Technology Transfer under the Clean Development Mechanism. Schriftenreihe des IÖW 175/04, Berlin 2004. \\ The People's Republic of China: Autorised Release: The Renewable Energy Law. Peking 2005. Download www.renewableenergyaccess.com/ assets/download/China_RE_Law_05.doc}

I AUTOR + KONTAKT

Ulrich Oberndorfer ist Diplomand an der Freien Universität Berlin und studentische Hilfskraft am Institut für ökologische Wirtschaftsforschung (IÖW).

IÖW, Potsdamer Str. 105, 10785 Berlin. E-Mail: ulrich.oberndorfer@ioew.de 
(c) 20I0 Authors; licensee IÖW and oekom verlag. This is an article distributed under the terms of the Creative Commons Attribution Non-Commercial No Derivates License (http://creativecommons.org/licenses/by-nc-nd/3.o/), which permits unrestricted use, distribution, and reproduction in any medium, provided the original work is properly cited. 